\title{
Indications for the use of highest priority critically important antimicrobials in the veterinary sector
}

\author{
Guillaume Lhermie ${ }^{10}{ }^{1,2 *}$, Roberto Marcello La Ragione ${ }^{3}$, J. Scott Weese ${ }^{4}$, John Elmerdahl Olsen ${ }^{5}$, \\ Jens Peter Christensen ${ }^{5}$ and Luca Guardabassi ${ }^{5,6}$ on behalf of the ESCMID Study Group for Veterinary \\ Microbiology (ESGVM)
}

${ }^{1}$ Department of Population Medicine and Diagnostic Sciences, College of Veterinary Medicine, Cornell University, Ithaca, NY, USA; ${ }^{2} I H A P$, Université de Toulouse, INRA, ENVT, Toulouse, France; ${ }^{3}$ Department of Pathology and Infectious Diseases, School of Veterinary Medicine, Faculty of Health and Medical Sciences, University of Surrey, Guildford GU2 7AL, UK; 'Department of Pathobiology, Ontario Veterinary College, University of Guelph, Guelph, Ontario, Canada; Department of Veterinary and Animal Sciences, Faculty of Health and Medical Sciences, University of Copenhagen, 1870 Frederiksberg C, Denmark; 'Department of Pathobiology \& Population Sciences, Royal Veterinary College, Hawkhead Lane, North Mymms, Hatfield, Herts AL9 7TA, UK

\author{
*Corresponding author. E-mail: g1467@cornell.edu
}

\begin{abstract}
Background: Among the measures taken to preserve the clinical efficacy of highest priority critically important antimicrobials (HP-CIAs), the WHO has recommended avoiding their use in food-producing animals. Little is known regarding the indications for which different antimicrobial classes are used in animals, even in countries where data on antimicrobial use are available.
\end{abstract}

Objectives: To outline, in a narrative review, the diseases for which HP-CIAs are used in veterinary medicine, highlighting incongruences with international guidelines and disease conditions where effective alternatives to HP-CIAs are missing.

\begin{abstract}
Methods: Scientific literature, national reports and expert opinion were used to describe the indications for the use of HP-CIAs in the main food-producing (pigs, cattle and poultry) and companion (horses, dogs and cats) animal species.
\end{abstract}

\begin{abstract}
Results: The most common indications for use of HP-CIAs are enteric and respiratory infections in pigs, cattle and poultry, urogenital infections in dogs and cats and respiratory infections in horses. In some instances, no valid and convenient alternatives to colistin and macrolides are available against certain porcine enteric and bovine respiratory pathogens. Effective, legal and convenient alternatives to HP-CIAs are also lacking for managing common infections in cats, for which oral administration is difficult, Rhodococcus equi infections in horses, some enteric and respiratory infections in poultry and MDR infections in all companion animal species.
\end{abstract}

Conclusions: Future research and stewardship programmes should focus on the disease conditions identified by this review to reduce the use of HP-CIAs in the veterinary sector.

\section{Introduction}

Humans are exposed to antimicrobial-resistant bacteria and resistance determinants of animal origin through the consumption of animal food products ${ }^{1}$ and via direct or indirect contact with livestock $^{2}$ and companion animals. ${ }^{3}$ The recent spread in animals of MDR bacteria of clinical relevance in human medicine has raised public health concerns about the use of certain antimicrobials in the veterinary sector. ${ }^{4}$ As a global public health threat, antimicrobial resistance has led national and international organizations to design and implement policies aimed at preserving the efficacy of medically important antimicrobials. The WHO has classified five classes of highest priority critically important antimicrobials
(HP-CIAs) based on their clinical importance in human medicine and the risk of resistance transfer from animals: third-, fourthand fifth-generation cephalosporins (hereafter referred to as extended-spectrum cephalosporins), macrolides and ketolides, glycopeptides, polymyxins and quinolones. ${ }^{5}$ In early 2018 , the WHO released guidelines to preserve the effectiveness of medically important antimicrobials; in these guidelines it is recommended that drugs classified as HP-CIAs for human medicine should not be used for the treatment of food-producing animals with clinically diagnosed infectious disease. ${ }^{6}$ In 2019, the Antimicrobial Advice ad hoc Expert Group (AMEG), established by the EMA, recommended that these HP-CIAs, with the exception of macrolides, 
should be considered only for the treatment of clinical conditions when there are no alternative antimicrobials of lower medical importance. $^{7}$

Data on antimicrobial consumption are only available for a limited number of countries and geographical areas, such as the EU. In 2017, the quantity of extended-spectrum cephalosporins used in European livestock production, measured in milligrams of active ingredient sold per population correction unit (mg/PCU), was relatively low $(0.2 \mathrm{mg} / \mathrm{PCU})$ in comparison with the quantity of fluoroquinolones (2.4 mg/PCU), polymyxins $(3.6 \mathrm{mg} / \mathrm{PCU})$ and macrolides (7.1 mg/PCU). ${ }^{8}$ Notable variations were observed in the consumption of HP-CIAs between countries $(<0.01$ to $0.8 \mathrm{mg} / \mathrm{PCU}$ for extended-spectrum cephalosporins, $<0.01$ to $15.3 \mathrm{mg} / \mathrm{PCU}$ for fluoroquinolones, 0 to $14.9 \mathrm{mg} / \mathrm{PCU}$ for polymyxins and 0 to $22 \mathrm{mg} / \mathrm{PCU}$ for macrolides). However, even in countries where data on antimicrobial consumption are available, it is not possible to infer for what indications HP-CIAs are used in each animal species. This information is essential to identify the disease conditions that should be prioritized to minimize consumption of these antimicrobials, for example by developing disease-specific clinical practice guidelines for diagnosis, management and treatment, or by incentivizing research on vaccines and non-antibiotic alternatives for the prevention and control of those disease conditions.

The aim of this narrative review is to outline the diseases for which HP-CIAs are used in the most important animal species used for terrestrial livestock production (pigs, cattle and poultry) and companionship (dogs, cats and horses), highlighting specific disease conditions where effective alternatives to HP-CIAs are lacking. Aquatic animals were not included in this review, as the biomass of terrestrial animals for which data are available and allowing some comparisons by far exceeds the biomass of aquatic food-producing animals. ${ }^{9}$ Furthermore, according to the 2017 World Organisation for Animal Health (OIE) report, aquatic food-producing animals are mainly treated with noncritical antimicrobials, such as amphenicols (65\%) and tetracyclines (23.5\%). ${ }^{10}$

\section{Methods}

We identified references for this review through searches of MEDLINE/ PubMed, EMBASE and Google Scholar. Search terms used for literature published in PubMed included (cattle), (cow), (bovine), (pig), (poultry), (layer), (broiler), (equine), (horse), (companion animal), (dog), (cat), (highest priority critically important antimicrobials), (macrolides), (ketolides), (cephalosporins), (fluoroquinolones), (polymyxins) and (glycopeptides). We also manually searched citations contained in the reference lists of articles and reports important for the purpose of the review. Twenty articles and reports focusing on HP-CIAs or presenting data on HP-CIA use among other classes were included in this review: eight covering multiple species, three in pigs, three in cattle, one in horses and five in companion animals (Table 1). The different metrics used to report usage of HP-CIAs are summarized in Table 2. During the revision process, the manuscript was circulated twice among the members of the ESCMID Study Group for Veterinary Microbiology (ESGVM) to gather feedback from other experts.

\section{Antimicrobial use in pigs}

Four groups of HP-CIAs are used in pig production: extendedspectrum cephalosporins, macrolides, fluoroquinolones and

Table 1. Year, country, reported metric, category of data, quality of evidence and type of collection for each of the references used for presenting data on the use of HP-CIAs

\begin{tabular}{|c|c|c|c|c|c|c|}
\hline $\begin{array}{l}\text { Reference } \\
\text { number }\end{array}$ & $\begin{array}{l}\text { Years of } \\
\text { collection }\end{array}$ & Country & Main metric reported & $\begin{array}{l}\text { Category } \\
\text { of data }\end{array}$ & $\begin{array}{l}\text { Quality of evidence } \\
\text { (type of sample) }\end{array}$ & $\begin{array}{l}\text { Type of } \\
\text { collection }\end{array}$ \\
\hline 8 & 2016-17 & multiple & $\mathrm{mg} / \mathrm{PCU}$ & global level & mandatory & cross-sectional \\
\hline 12 & 2015 & Vietnam & $\mathrm{mg} / \mathrm{PCU}$ & country level & convenience & cross-sectional \\
\hline 13 & 2012 & multiple & percentage of use & regional level & convenience & cross-sectional \\
\hline 14 & 2012 & Sweden & DADD & country level & mandatory & cross-sectional \\
\hline 15 & 2013-15 & Germany & DADD/half year/farm & country level & mandatory & longitudinal \\
\hline 16 & $2008-17$ & France & ALEA & country level & mandatory & longitudinal \\
\hline 18 & 2015 & Denmark & DDDA per 1000 pig-days & country level & mandatory & cross-sectional \\
\hline 19 & 2014 & Netherlands & tons & country level & mandatory & cross-sectional \\
\hline 24 & 2010-15 & EU & $\mathrm{mg} / \mathrm{PCU}$ & country level & mandatory & longitudinal \\
\hline 34 & 2016 & USA & tons & country level & mandatory & cross-sectional \\
\hline 35 & 2003-15 & Ireland & DDDvet/cow/year & country level & convenience & longitudinal \\
\hline 38 & $2012-16$ & Netherlands & DDDvet/PCU & country level & mandatory & longitudinal \\
\hline 40 & 2011 & USA & percentage of use & country level & convenience & cross-sectional \\
\hline 42 & 2014 & Denmark & $\mathrm{ADD}$ & country level & convenience & longitudinal \\
\hline 54 & $2012-14$ & UK & percentage of use & country level & convenience & longitudinal \\
\hline 55 & $2012-13$ & UK & percentage of use & country level & convenience & longitudinal \\
\hline 56 & $2014-16$ & UK & percentage of use & country level & convenience & longitudinal \\
\hline 58 & 2011 & Canada & percentage of use & country level & convenience & longitudinal \\
\hline 59 & 2000-07 & Italy & percentage of use & country level & convenience & longitudinal \\
\hline 65 & 2011 & UK & percentage of use & country level & convenience & cross-sectional \\
\hline
\end{tabular}

DADD, defined animal daily dose; ALEA, animal level of exposure to antimicrobials; DDDA, DDD animal; ADD, animal daily dose; DDDvet, defined daily dose for animals. 
Table 2. Description of the metrics used in the selected references

\begin{tabular}{|c|c|c|c|}
\hline Metric & & Calculation & Comment \\
\hline $\begin{array}{l}\text { Amount of active } \\
\text { ingredient }\end{array}$ & $\begin{array}{l}\text { numerator: } \\
\text { denominator: }\end{array}$ & $\begin{array}{l}\text { active ingredient weight (kg or tons) } \\
\text { none }\end{array}$ & amount of active ingredient sold \\
\hline No. of mg/PCU & $\begin{array}{l}\text { numerator: } \\
\text { denominator: }\end{array}$ & $\begin{array}{l}\text { active ingredient weight }(\mathrm{mg}) \\
\text { standardized average weight }(\mathrm{kg}) \text { of all animals at time of } \\
\text { treatment } \times \text { total no. of animals based on national } \\
\text { statistics (live and/or slaughter) }\end{array}$ & $\begin{array}{l}\text { amount of active ingredient } \\
\text { per biomass at risk }\end{array}$ \\
\hline ALEA & $\begin{array}{l}\text { numerator: } \\
\text { denominator: }\end{array}$ & $\begin{array}{l}\text { weight of animals treated }(\mathrm{kg}) \\
\text { no. of animals treated } \times \text { weight at slaughter }(\mathrm{kg})\end{array}$ & biomass at risk of being treated \\
\hline No. of DDDA & $\begin{array}{l}\text { numerator: } \\
\text { denominator: }\end{array}$ & $\begin{array}{l}\text { average daily dose }(\mathrm{mg} / \mathrm{kg}) \times \text { weight at slaughter }(\mathrm{kg}) \\
\text { none }\end{array}$ & no. of animals treated \\
\hline No. of DDDvet & $\begin{array}{l}\text { numerator: } \\
\text { denominator: }\end{array}$ & $\begin{array}{l}\text { amount of active ingredient }(\mathrm{mg}) \\
\text { DDDvet for the active ingredient }\end{array}$ & no. of $\mathrm{kg}$ per day being treated \\
\hline No. of DDDvet/PCU & $\begin{array}{l}\text { numerator: } \\
\text { denominator: }\end{array}$ & $\begin{array}{l}\text { no. of DDDvet } \\
\text { PCU (see calculation above) }\end{array}$ & no. of treatments per year \\
\hline DDDA per 1000 pig-days & $\begin{array}{l}\text { numerator: } \\
\text { denominator: }\end{array}$ & $\begin{array}{l}\text { no. of DDDA } \times 365 \times 1000 \\
\text { production days } \times \text { standardized liveweight }\end{array}$ & no. of treatments per year \\
\hline DADD/half year/farm & $\begin{array}{l}\text { numerator: } \\
\text { denominator: }\end{array}$ & $\begin{array}{l}\text { no. of DADD } \\
\text { none }\end{array}$ & no. of treatments per half year \\
\hline No. of DDDvet/cow/year & $\begin{array}{l}\text { numerator: } \\
\text { denominator: }\end{array}$ & $\begin{array}{l}\text { no. of DDDvet } \times 365 \\
\text { production days } \times \text { standardized liveweight }\end{array}$ & no. of treatments per year \\
\hline DADD & $\begin{array}{l}\text { numerator: } \\
\text { denominator: }\end{array}$ & $\begin{array}{l}\text { total amount of active ingredient } \\
\text { DDDvet } \times 365\end{array}$ & no. of animals treated \\
\hline TI ADD & $\begin{array}{l}\text { numerator: } \\
\text { denominator: }\end{array}$ & $\begin{array}{l}\text { total amount of active ingredient/(DDDvet } \times \text { production } \\
\text { days } \times \text { standardized liveweight) } \\
\text { no. of animals at risk of being treated } \times 1000\end{array}$ & no. of animals at risk of being treated \\
\hline
\end{tabular}

DADD, defined animal daily dose; ALEA, animal level of exposure to antimicrobials; DDDA, DDD animal; ADD, animal daily dose; DDDvet, defined daily dose for animals; TI ADD, Treatment Incidence based on ADD.

polymyxins (colistin). The glycopeptide avoparcin was commonly used for growth promotion in the 1990s, mainly in Europe and Australia, but concerns regarding the selection of VRE led to a ban on the use of this drug in food animals in the EU and later in Australia, Japan and China. The drug has never been approved for food animals in the USA. ${ }^{11}$ A recent survey on the use of antimicrobials in pig production in Vietnam did not find avoparcin in feed for pigs. ${ }^{12}$ Owing to the lack of regulation and reliable data on antimicrobial consumption at the global level, it cannot be excluded that this glycopeptide may still be in use in some countries for prophylactic, therapeutic or growth-promotion purposes.

According to a European survey among pig practitioners, ${ }^{13} \mathrm{HP}$ CIAs are mainly used for treatment of diarrhoea (64\%, including $30 \%$ polymyxins, $22 \%$ macrolides and $12 \%$ fluoroquinolones), followed by postpartum dysgalactia syndrome (31\%, including $21 \%$ fluoroquinolones and $7 \%$ extended-spectrum cephalosporins), respiratory diseases (12\%, including $10 \%$ macrolides) and Streptococcus suis infections (5\%, including 3\% extendedspectrum cephalosporins). There are, however, significant differences between European countries in the use of HP-CIAs in pig production. ${ }^{14}$ Within each country, consumption of HP-CIAs can vary over time because of changes in disease occurrence and national policies, unfortunately not always in the desired direction. For example, a dramatic increase in the use of ceftiofur for the treatment of suckling pigs was observed in Germany between
2013 and $2015,{ }^{15}$ followed by a marked reduction in the following years due to restrictions in the prescription of HP-CIAs. Similar policies have led to a significant reduction in the use of extendedspectrum cephalosporins, fluoroquinolones and colistin in pig production in other EU countries such as France and Sweden. . $^{16,17}$ In the Netherlands and Denmark, pig producers have restricted the use of these antimicrobials on a voluntary basis. ${ }^{18,19}$ It is noteworthy that pig production in Denmark has shown an increasing trend over time, ${ }^{20}$ suggesting that such restrictions do not affect pig productivity. In Belgium, treatment guidelines advocating strict regulation of the use of HP-CIAs were published by the Center of Expertise on Antimicrobial Consumption and Resistance in Animals in 2013 and this country has also succeeded in a significant reduction in the use of HP-CIAs. ${ }^{21}$ Together, these data suggest that once stakeholders agree on reduction in HP-CIA use in pig production, this can be achieved in a relatively short time. Follow-up studies on reduction strategies in Denmark indicate that farmers adapt to restrictions in the use of antimicrobials by changing management strategies, including decision strategies for treatment. ${ }^{22}$

Colistin constitutes a special case. For many years it was only used in veterinary medicine owing to toxicity in humans but in recent years it has become a last-resort drug for treatment of sepsis and pneumonia caused by XDR Gram-negative bacteria in humans. ${ }^{23}$ For this reason, colistin was reclassified as an HP-CIA in the most recent edition of the WHO classification. ${ }^{6}$ Its use in 
livestock appears to have declined in several countries following a recommendation by the EMA. ${ }^{24}$ This polymyxin is widely used as a feed additive in pigs, especially in low- and middle-income countries. ${ }^{12,25}$ It is a drug of choice for treatment of Escherichia coli diarrhoea, since resistance to other veterinary antimicrobials is common in this species, and thus frequently used for this indication.

Zinc oxide is the only non-HP-CIA alternative to colistin for managing gastrointestinal infections caused by $E$. coli strains resistant to aminopenicillins, tetracyclines and aminoglycosides. Zinc oxide is not regarded as an HP-CIA because it is not used for systemic antimicrobial therapy in human medicine. However, its use in livestock production has ecological implications associated with environmental pollution and, above all, has been shown to co-select for MRSA. ${ }^{26-28}$ Based on these potential hazards to public health, the European Commission has called for a withdrawal of marketing authorization for veterinary products containing zinc oxide to be effective by $2022 .{ }^{29}$ Possible alternatives include aminoglycosides (e.g. neomycin) and amoxicillin/clavulanate, which are grouped in the same category (C) as macrolides in the recent AMEG classification based on criticality of use in human medicine and probability of spread of resistance from animals to humans, and should only be used when there are no effective antimicrobials in the lowest category (D). ${ }^{7}$ So, safe and effective antimicrobial alternatives to colistin are lacking for this indication.

As for the macrolides, they are classified as HP-CIAs by the WHO because there are limited therapeutic options for treatment of Legionella, Campylobacter and MDR Salmonella and Shigella infections in human medicine and their use in animals may facilitate transmission of macrolide-resistant Campylobacter and Salmonella to humans. ${ }^{6}$ However, macrolides are also critically important in porcine veterinary practice as they are widely used to control common infections such as enteritis, respiratory infections and arthritis. For example, tylosin is mainly used in medicated feed for treatment and prevention of porcine proliferative enteropathy, swine dysentery and respiratory disease, whereas injectable macrolides (e.g. tilmicosin and tulathromycin) are indicated for control of enzootic pneumonia and other respiratory infections. ${ }^{30}$ They constitute $12 \%$ of total antimicrobials used in Danish pig production (66\% in weaned pigs) and approximately $35 \%$ of herd treatments in Swedish pig production. ${ }^{18}$ Tiamulin and lincosamides can be used as valid alternatives to macrolides for Lawsonia and Brachyspira enteric infections and for Mycoplasma arthritis. The pan-European antimicrobial resistance monitoring programme VetPath reported absence or low prevalence $(<2 \%)$ of resistance to non-HP-CIA alternatives to macrolides in pig respiratory pathogens, namely amoxicillin/clavulanate, florfenicol and tiamulin. ${ }^{31}$ Similarly, relatively low levels of resistance have also been reported in Australia, ${ }^{32}$ suggesting that it would not be a problem to replace macrolides for treatment of porcine respiratory infections in these geographical areas. Macrolides are also widely used for treatment of porcine enzootic pneumonia associated with Mycoplasma hyopneumoniae. Clinical breakpoint values do not exist for this organism. However, a study based on a geographically wide strain collection has shown low $\mathrm{MIC}_{50} / \mathrm{MIC}_{90}$ values for tulathromycin $(\leq 0.001 / 0.004 \mathrm{mg} / \mathrm{L})$ and tylosin $(0.031 / 0.125 \mathrm{mg} / \mathrm{L})$, but also for non-HP-CIAs such as florfenicol $(0.25 / 0.5 \mathrm{mg} / \mathrm{L})$ and oxytetracycline $(0.062 / 0.25 \mathrm{mg} / \mathrm{L}){ }^{33}$ Thus, it appears that macrolides are not essential medicines for managing porcine respiratory infections.
Their widespread use for this indication is probably attributable to the convenience of administering long-acting formulations, which are not yet available for other antimicrobial classes.

\section{Antimicrobial use in cattle}

The HP-CIAs used in cattle production are the same as for pig production. As for the other animal species, global data on antimicrobial use in cattle are crude and scarce, arising mainly from Western countries. Sales data show that cattle accounted for $43 \%$ of the mass of medically important antimicrobials used in the USA in 2016 (as listed in the FDA's Guidance for Industry \#152). ${ }^{34}$ European veterinarians are reported to use HP-CIAs mainly for treatment of diarrhoea $(69 \%$, including $40 \%$ polymyxins, $20 \%$ fluoroquinolones and $8 \%$ extended-spectrum cephalosporins), followed by respiratory diseases ( $44 \%$, including $27 \%$ macrolides and $13 \%$ fluoroquinolones), lameness (31\%, including $22 \%$ extended-spectrum cephalosporins and $9 \%$ macrolides), mastitis (22\%, including $11 \%$ extended-spectrum cephalosporins and $6 \%$ macrolides) and metritis (21\%, including $18 \%$ extended-spectrum cephalosporins). ${ }^{13}$ Colistin is not available in all countries but, where available, remains an antimicrobial of choice for the control of $E$. coli diarrhoea in calves, with few antimicrobial alternatives depending on the susceptibility profile of the causative strain. However, the use of other HP-CIAs is difficult to justify on the basis of antimicrobial resistance patterns in cattle pathogens.

The diseases for which HP-CIAs are used in cattle vary depending on the type of production (dairy, beef and veal calf production). In dairy production, antimicrobials can be administered locally via intramammary treatment or systemically for control of clinical and subclinical mastitis. Intramammary formulations are used for treatment during lactation or for prophylaxis at dry-off. The most common HP-CIAs for this route of administration are extendedspectrum cephalosporins (ceftiofur and cefquinome); these, however, account for a low proportion of intramammary treatments in EU countries. For example, they represented $1.6 \%$ of the number of intramammary applications in Denmark in $2016^{18}$ and $1.7 \%$ of the total of active substances sold for intramammary treatment in Ireland in 2015 (6.9\% and 5.4\% of the products for lactation and drying-off treatment, respectively). ${ }^{35}$ Based on VetPath, mastitis pathogens are usually susceptible to most antibiotics. ${ }^{36}$ Thus, the use of extended-spectrum cephalosporins for this indication is not justified by resistance to antimicrobials of lower medical importance. Altogether, these data indicate that extended-spectrum cephalosporins are not critically important for intramammary treatment of mastitis. Extended-spectrum cephalosporins are also indicated for parenteral treatment of lameness, mastitis, metritis and respiratory disease. Depending on the countries and the pathogen involved, one or several alternative treatments with non-HP-CIAs are available for these indications. However, some cephalosporin formulations (e.g. ceftiofur) are attractive, not only for their effectiveness, but also for their convenience of use as well as the short withdrawal period during which milk cannot be sold.

The efficacy of fluoroquinolones has been shown for systemic treatment of severe clinical mastitis due to Gram-negative pathogens. ${ }^{37}$ However, it is noteworthy that such severe mastitis only represents a minority of clinical cases. The vast majority of E. coli and Klebsiella isolates from mastitis are susceptible to alternative antimicrobials such as amoxicillin/clavulanate, first-generation 
cephalosporins and aminoglycosides, ${ }^{36}$ indicating that the essentiality of fluoroquinolones in dairy production is questionable on the basis of microbiological data. This is also confirmed by the fact that dairy cattle are produced efficiently with a consumption of fluoroquinolones close to zero in countries such as Denmark and the Netherlands. ${ }^{18,38}$ This goal was accomplished by regulatory measures restricting the use of fluoroquinolones to last-resort drugs, only when justified by microbiological analysis and susceptibility testing in an accredited laboratory. The implementation of similar rules for the prescription of fluoroquinolones led to a drastic reduction $(75 \%)$ in the usage of these antimicrobials in French cattle between 2013 and $2016 .{ }^{16}$ Because of the absence of market authorization, fluoroquinolones are not used for the treatment of lactating cows in North America.

Bovine respiratory disease (BRD) is the most frequently occurring disease that requires antimicrobial treatment in beef and veal calf production. The use of antimicrobials for this indication is particularly high in the feedlot system, which is common in North America. ${ }^{39}$ Surveys conducted in the USA in 2011 showed that $45 \%, 16 \%$ and $10 \%$ of the US cattle were treated with macrolides, fluoroquinolones and extended-spectrum cephalosporins, respectively, and that most of the treatments were given as prophylaxis or metaphylaxis. ${ }^{40}$ Metaphylaxis executed during the incubation period of the disease is a well-documented way to decrease morbidity and mortality due to BRD in high-risk cattle. ${ }^{40}$ Macrolides are extensively used because they are highly effective in preventing and controlling BRD outbreaks, ${ }^{41}$ but also because their long-acting formulation allows them to cover approximately the length of the period at the beginning of the fattening process, during which young cattle are more likely to become sick.

Liver abscesses are infections due to anaerobic bacteria, affecting $10 \%$ to $20 \%$ in most feedlots. Cattle presenting abscessed livers had a reduced weight gain and carcass dressing percentage and a lower feed efficiency. Even though other antimicrobials are approved, in-feed use of tylosin is effective and frequent for the prevention of liver abscesses. ${ }^{42}$

Overall, decreasing the use of HP-CIAs in cattle production is possible, with the magnitude of reduction highly dependent on the baseline level of use and the production system. The segmented infrastructure of the feedlot and veal calf industries favours antimicrobial use because these animals are transported several times and exposed to multiple environmental and nutritional changes and other stress factors that predispose to diseases such as BRD. ${ }^{43}$ Therefore, a significant reduction in antimicrobial usage in these production systems, including the use of HP-CIAs, would require in-depth structural and behavioural changes in the market.

\section{Antimicrobial use in poultry}

The most widely used HP-CIAs in poultry production include enrofloxacin, colistin and macrolides. The use of these HP-CIAs varies considerably from country to country, depending on local guidelines and legislation. Usually, one or several alternative treatments with non-HP-CIAs are available for most (respiratory, enteric and systemic) infections in both broiler and layer production.

Enrofloxacin is often used for treatment of broiler parent flocks, mainly for extraintestinal (avian pathogenic E. coli) respiratory or systemic infections. It is also indicated for the treatment of respiratory and intestinal tract infections in broilers caused by
Mycoplasma gallisepticum, Mycoplasma synoviae, Avibacterium gallinarum, Pasteurella multocida, Salmonella and E. coli. ${ }^{44}$ Although alternatives to enrofloxacin exist for Gram-negative infections (e.g. ampicillin, amoxicillin, tetracyclines and sulphonamides), they are not as effective. ${ }^{18}$ However, the use of fluoroquinolones is illegal for off-label use in the USA and very restricted in other countries (e.g. in northern Europe), suggesting that broilers can be produced with no or minimal use of enrofloxacin. Similarly, colistin is mainly used to treat Gram-negative enteric and respiratory infections in broilers, but many countries have either banned or restricted its use, showing it cannot be regarded as an essential antibiotic for poultry production.

In broiler production, macrolides such as tylosin, tilmicosin and erythromycin are used to treat infections due to Mycoplasma and Ornithobacterium rhinotracheale (ORT), P. multocida and sometimes Clostridium perfringens (necrotic enteritis). Suitable, effective alternatives to the macrolides to treat necrotic enteritis and fowl cholera (P. multocida) are penicillin $\mathrm{G}$ and doxycycline/oxytetracycline. ${ }^{45}$ An alternative to the macrolides for the treatment of Mycoplasma infections is more difficult to recommend as they are clearly the best choice, but tetracyclines may be of value. ${ }^{46}$ In layers, macrolides are mainly used to treat infections caused by M. gallisepticum, M. synoviae, Staphylococcus aureus, O. rhinotracheale, Brachyspira and C. perfringens. ${ }^{47}$ The same alternatives to these HP-CIAs recommended for broilers may be used in layers but poorer effectiveness may be observed. ${ }^{48}$

Extended-spectrum cephalosporins are less widely used in poultry production. In the EU, because of the lack of established maximum residue limits, no label indications exist for the use of ceftiofur in poultry and off-label use is discouraged. ${ }^{49}$ In the past, ceftiofur has been extensively used off-label by in ovo administration to prevent contamination of hatching eggs and contamination from vaccination procedures. ${ }^{50}$ In some countries, extended-spectrum cephalosporins are still used in the hatchery for prevention of E. coli infections in day-old chicks, although their use is restricted in many countries. ${ }^{45}$ Nevertheless, it should be highlighted that extended-spectrum cephalosporins are not essential for any indications in poultry production.

\section{Antimicrobial use in dogs and cats}

Among the antimicrobials classified as HP-CIAs by the WHO, fluoroquinolones (e.g. enrofloxacin, ciprofloxacin, marbofloxacin and pradofloxacin) and extended-spectrum cephalosporins (e.g. cefovecin and, limited to some countries, cefpodoxime) are the most frequently prescribed in small animal veterinary practice. According to a survey among veterinarians in Europe, ${ }^{13}$ use of HPCIAs is most frequent for urogenital infections in both dogs $(27 \%$, including $26 \%$ fluoroquinolones) and cats (62\%, including $49 \%$ fluoroquinolones and $13 \%$ cephalosporins). Other indications for which these antimicrobials are prescribed include respiratory (16\%, including $13 \%$ fluoroquinolones), skin (10\%, including $9 \%$ fluoroquinolones) and gastrointestinal disease $(7 \%$, including $5 \%$ fluoroquinolones) in dogs; and periodontal (38\%, including $21 \%$ cephalosporins and $14 \%$ macrolides), skin (24\%, including $16 \%$ cephalosporins and $8 \%$ fluoroquinolones) and respiratory disease (16\%, including $11 \%$ fluoroquinolones and $3 \%$ cephalosporins) in cats. These prescription patterns are in contrast with the clinical practice guidelines for skin, urinary tract and respiratory 
infections ${ }^{51-53}$ since both fluoroquinolones and extendedspectrum cephalosporins are generally classified as second- or third-line agents, except for severe life-threatening infections (e.g. sepsis and severe lower respiratory tract infections) or rare situations where fluoroquinolones outcompete other antimicrobial classes in terms of clinical efficacy and safety (e.g. prostatitis or pyelonephritis). The prescribing behaviour of primary care veterinary practitioners is often not in agreement with national guidelines either, as indicated by a recent survey in Belgium showing that bacterial culture and antimicrobial susceptibility testing are only used in $12.4 \%$ of the HP-CIA prescriptions. ${ }^{54}$

Various studies report usage of HP-CIAs expressed as percentages of antimicrobial prescriptions. ${ }^{54-57}$ According to a large survey based on clinical records from 963463 dogs and 594812 cats in the UK, ${ }^{55} \mathrm{HP}$-CIAs accounted for just over $6 \%$ and $34 \%$ of antimicrobial events in dogs and cats, respectively. A similar study among Belgian primary care practitioners reported approximately $15 \%$ and $11 \%$ prescriptions for fluoroquinolones and extended-spectrum cephalosporins, respectively. ${ }^{54}$ In both studies, fluoroquinolones and extended-spectrum cephalosporins were the most commonly used HP-CIAs in dogs and cats, respectively. The higher usage of extended-spectrum cephalosporins reflected the more frequent use of cefovecin in cats ( $54 \%$ of total cat events versus $1 \%$ of dog events). Cefovecin is a long-acting third-generation cephalosporin that requires a single subcutaneous administration, covering a course of treatment (for some pathogens and body sites) of 14 days, making this drug very attractive for cats, for which oral administration can be difficult in some animals. Because of the convenience, this drug may be prone to overuse. A recent study in the UK identified skin (48\%), urinary tract $(14 \%)$ and respiratory infections $(10 \%)$ as the most common cat disease conditions for which cefovecin is prescribed in first-opinion practices. ${ }^{56}$ Another study based on 218 community small animal practices in Ontario, Canada, revealed a likely overuse of extended-spectrum cephalosporins and fluoroquinolones for the treatment of feline upper respiratory tract disease, feline lower urinary tract disease and canine infectious respiratory disease complex. ${ }^{58}$ Together the two antimicrobial classes accounted for $12 \%$ and $29 \%$ of all antimicrobial prescriptions in dogs and cats, respectively. Fluoroquinolones are particularly used for management of urinary tract infections, as indicated by an Italian study, where use of this antimicrobial class exceeded $20 \%$ of the total prescriptions and $60 \%$ of the prescriptions for urinary tract infections in a veterinary teaching hospital. ${ }^{59}$ Overuse of fluoroquinolones in dogs for management of acute gastroenteritis (26\% of all prescriptions for this indication), uncomplicated urinary tract infections (22\%) and acute tracheobronchitis (15\%) was reported by a recent survey among primary care practitioners in Belgium. ${ }^{54}$ The same study also documented an excessive use of extended-spectrum cephalosporins for treatment of skin infections $(25 \%)$ and upper respiratory tract disease $(11 \%)$ in cats. As with cefovecin use in cats, the convenience of once-daily dosing of fluoroquinolones and the third-generation cephalosporin cefpodoxime may explain the frequent use of these drug classes in dogs.

As for the remaining HP-CIAs, macrolides are not commonly used in dogs and cats owing to side effects, although azithromycin is increasingly used for the management of upper respiratory tract, dental and periodontal disease. The recent International Society for Companion Animal Infectious Diseases (ISCAID) guidelines for respiratory infections promote prudent use of azithromycin by recommending doxycycline as a first choice and no antimicrobial treatment during the first 10 days after the appearance of clinical signs. ${ }^{53}$ Ketolides and polymyxins (except topical eye and ear drops) are not used, whereas glycopeptides are very rarely used for management of Corynebacterium urealyticum encrusting cystitis $^{60}$ or severe methicillin-resistant staphylococcal infections that require systemic treatment and are not otherwise treatable. ${ }^{61}$ Some MDR infections cannot be managed using antimicrobials authorized for veterinary use, e.g. urinary tract infections caused by MDR E. coli and deep pyoderma associated with methicillinresistant Staphylococcus pseudintermedius (MRSP). Alternatives for treatment of these infections are needed to avoid extra-label use of last-choice antimicrobials in human medicine (e.g. glycopeptides and linezolid against MRSP and carbapenems against Enterobacteriaceae). Use of the latter drugs has been banned in some countries (e.g. Sweden) and in other countries it is only justified in rare and otherwise untreatable cases characterized by animal discomfort and high social or economic value of the individual patient and where lack of treatment may have important ethical and legal implications for the veterinarian. ${ }^{62}$

\section{Antimicrobial use in horses}

Extended-spectrum cephalosporins, and to a lesser extent fluoroquinolones, are the most commonly used HP-CIAs. Glycopeptides are rarely used, polymyxins can be used in the treatment of colic and macrolides are only used for specific indications, although they are of critical importance clinically in those situations, particularly the management of Rhodococcus equi infections, where they are the best investigated and most efficacious options. While erythromycin has been the main macrolide used in the past, newer drugs including azithromycin, clarithromycin, tulathromycin and gamithromycin are also used.63,64

According to a large-scale survey among equine veterinary practitioners in Europe, ${ }^{13}$ use of HP-CIAs was reported more frequently for respiratory disease (18\%, including 13\% cephalosporins) than for locomotive disorders (9\%, including $7 \%$ cephalosporins), perioperative prophylaxis (6\%, including $4 \%$ cephalosporins and $2 \%$ fluoroquinolones) and skin diseases (4\%, including 3\% cephalosporins). Although horses can encounter various bacterial pathogens, viral disease and non-infectious aetiologies are common, but may lead to antimicrobial treatment and overuse of HP-CIAs. A case scenario-based study of UK veterinarians revealed a high likelihood of antimicrobial prescription for pyrexic yearlings (where viral infection is most likely) and coughing adult horses (where inflammatory airway disease is most likely). ${ }^{65}$ Ceftiofur, cefquinome and enrofloxacin were each recommended in $<3 \%$ of contaminated limb wounds, coughing pyrexic yearlings, coughing adult horses and pastern dermatitis scenarios. Higher rates of use of HP-CIAs were identified in a survey of German, Austrian and Swiss equine veterinarians, with extended-spectrum cephalosporins selected by $11 \%$ of veterinarians and fluoroquinolones by $4 \%{ }^{66}$ However, whether questionnaires and scenario situations reflect actual prescribing practices is unclear.

Emergence of MDR pathogens further complicates antimicrobial selection. MRSA is endemic in horses in many regions ${ }^{67-69}$ resulting in the loss of ability to use $\beta$-lactams and often other 
commonly used antimicrobials such as trimethoprim/sulphonamide and aminoglycosides. Other MDR pathogens such as ESBLproducing Enterobacteriaceae are also widely disseminated, with limited antimicrobial treatment options. ${ }^{70,71}$ Continued emergence and dissemination of MDR pathogens, and the increased risk of infection or colonization by these pathogens after antimicrobial exposure, highlight the potential risks posed by routine antimicrobial therapy and the potential for escalating use of HP-CIAs as MDR infections become more prevalent. ${ }^{71,72}$

\section{Concluding remarks}

Some European countries have shown that it is possible to raise healthy and productive livestock with minimal or no use of extended-spectrum cephalosporins and fluoroquinolones. However, with regard to the current situation of control of disease and availability of alternative treatments, total replacement of colistin and macrolides in pig and beef production is more difficult to achieve because some of the pathogens that cause substantial production losses have developed resistance to all of the drugs of lower medical importance. A notable example is MDR E. coli causing diarrhoea, which makes colistin clinically important for managing this economically impacting pathogen in pig and calf production. Similarly, macrolides are critically important for treatment of respiratory diseases in beef cattle, pigs, poultry and horses. At present, macrolides remain a better choice than fluoroquinolones or extended-spectrum cephalosporins, which are also indicated for treatment of respiratory infections, since the risks of zoonotic transfer of antimicrobial resistance appear to be lower. This is supported by the recent AMEG classification, where macrolides were included in a category at lower risk for human health. ${ }^{7}$

As for companion animals, the most commonly used HP-CIAs are fluoroquinolones and extended-spectrum cephalosporins, with use often in situations where alternative, lower-tier options are available. The use of these HP-CIAs can be reduced by implementing and updating the existing guidelines for superficial folliculitis and urinary tract and respiratory disease. Furthermore, in contrast with the situation in livestock, MDR infections are relatively frequent in companion animals and difficult to manage by on-label use of antimicrobials. For this reason, new veterinary-specific diagnostic and treatment strategies are needed for managing common MDR infections that require systemic treatment in small animal veterinary practice (e.g. urinary tract infections and deep pyoderma).

New veterinary-specific antimicrobial drugs characterized by high clinical efficacy and convenient administration would facilitate a reduction in the use of HP-CIAs. Long-acting formulations containing macrolides or extended-spectrum cephalosporins are widely used in livestock because of their short withdrawal periods and easy administration, which makes it possible to treat each diseased animal individually with reduced labour costs. A reduction in the use of such long-acting HP-CIAs could be achieved by developing similar formulations containing antimicrobials of lower medical importance. Similarly, alternative long-acting formulations would be useful to reduce the use of cefovecin in cats, since this drug is used more for the advantages of long-acting formulations (improved patient compliance and convenience for the owner and veterinarian) than for the need to use a third-generation cephalosporin.

Except for dogs and cats, international clinical practice guidelines are lacking for the most important disease conditions in veterinary medicine. Development and effective implementation of disease-specific guidelines is a cornerstone of antimicrobial stewardship and a well-known tool for improving antimicrobial use in human medicine. Clinical practice guidelines are particularly needed for the disease conditions that account for most use of HP-CIAs in livestock, namely enteric and respiratory disease in pigs and poultry, respiratory disease in poultry, beef and veal cattle and mastitis in dairy cow production. Disease-specific practice guidelines are also needed to reduce HP-CIA use for skin, respiratory and postsurgical infections in equine medicine and for enteric diseases in dogs and cats.

For all animal species, increased attention to infection prevention and control and biosecurity measures are critical, since prevention of disease eliminates the need for antimicrobials. In pig production, vaccines are available against some of the most important diseases in relation to antimicrobial use (postweaning diarrhoea and respiratory diseases), but they are not widely used, probably due to cost and limited efficacy in reducing antimicrobial use. ${ }^{73}$ No commercial vaccine is available against S. suis. In dairy cattle production, good vaccines against mastitis and metritis are lacking. Although several vaccines are available for both bacterial and viral infections in poultry and vaccines against Erysipelothrix, O. rhinotracheale, coryza and Pasteurella are widely used in poultry production, there is a need for new vaccines providing broad protection since the available ones are killed vaccines with no or little cross-protection against other serovars. Overall, developing effective vaccination programmes for controlling these diseases would likely have a significant impact on the use of HP-CIAs in livestock production.

Though beyond the scope of this paper, in which we mainly discussed the possibility of replacing HP-CIAs with non-critically important antimicrobials, it is worth mentioning that for several diseases, effective ways to curb use of HP-CIAs include the implementation of preventive measures, such as improved farm management, biosecurity and disease prevention. In some cases, alternatives to antimicrobial use already exist and have proven effective, such as the use of selective mastitis therapy in dairy production, preconditioning in beef production, or improved management and feeding practices in pig production. ${ }^{74-76}$ Although the panel of potential alternatives to antimicrobials has grown substantially in recent years, more research is needed to develop and validate effective and sustainable non-antimicrobial strategies, especially for managing the disease conditions identified by this review.

\section{Acknowledgements}

We are grateful to Julia Hertl (Cornell University) for manuscript editing.

\section{Transparency declarations}

None to declare.

\section{References}

1 Verraes C, Van Boxstael S, Van Meervenne E et al. Antimicrobial resistance in the food chain: a review. Int J Environ Res Public Health 2013; 10: 2643-69. 
2 Woolhouse M, Ward M, van Bunnik B et al. Antimicrobial resistance in humans, livestock and the wider environment. Philos Trans $R$ Soc Lond B Biol Sci 2015; 370: 20140083.

3 Pomba C, Rantala M, Greko C et al. Public health risk of antimicrobial resistance transfer from companion animals. J Antimicrob Chemother 2016; 72: 957-68.

4 Collignon P, Aarestrup FM, Irwin R et al. Human deaths and thirdgeneration cephalosporin use in poultry, Europe. Emerg Infect Dis 2013; 19: 1339-40.

5 WHO. Critically Important Antimicrobials for Human Medicine. 2017. http://apps.who.int/iris/bitstream/10665/255027/1/9789241512220-eng.pdf ?ua=1\%0Ahttp://www.oalib.com/references/1801489\%0Ahttp://apps.who. int/iris/bitstream/10665/77376/1/9789241504485_eng.pdf.

6 Aidara-Kane A, Angulo FJ, Conly JM et al. World Health Organization (WHO) guidelines on use of medically important antimicrobials in foodproducing animals. Antimicrob Resist Infect Control 2018; 7: 7.

7 EMA. Committee for Medicinal Products for Veterinary Use (CVMP). 2019. https://www.ema.europa.eu/en/documents/other/answer-request-euro pean-commission-updating-scientific-advice-impact-public-health-animalhealth-use_en.pdf.

8 EMA. Sales of Veterinary Antimicrobial Agents in 31 European Countries in 2017. Trends from 2010 to 2017. EMA/275982/2018 2019. https://www. ema.europa.eu/en/documents/report/sales-veterinary-antimicrobial-agents31-european-countries-2017_en.pdf.

9 Organisation for Economic Co-operation and Development, Food and Agriculture Organization of the United Nations. OECD-FAO Agricultural Outlook 2016-2025. Special Focus: Sub-Saharan Africa. 2016. http://www. fao.org/3/a-i5778e.pdf.

10 World Organisation for Animal Health (OIE). OIE Annual Report on Antimicrobial Agents Intended for Use in Animals. 2017. https://www.oie.int/ fileadmin/Home/eng/Our_scientific_expertise/docs/pdf/AMR/Annual_ Report_AMR_2.pdf.

11 Hao H, Sander P, Iqbal Z et al. The risk of some veterinary antimicrobial agents on public health associated with antimicrobial resistance and their molecular basis. Front Microbiol 2016; 7: 1626.

12 Van Cuong N, Nhung NT, Nghia NH et al. Antimicrobial consumption in medicated feeds in Vietnamese pig and poultry production. Ecohealth 2016; 13: 490-8.

13 De Briyne N, Atkinson J, Borriello SP et al. Antibiotics used most commonly to treat animals in Europe. Vet Rec 2014; 175: 325.

14 Sjölund M, Postma M, Collineau L et al. Quantitative and qualitative antimicrobial usage patterns in farrow-to-finish pig herds in Belgium, France, Germany and Sweden. Prev Vet Med 2016; 130: 41-50.

15 Schaekel F, May T, Seiler J et al. Antibiotic drug usage in pigs in Germany_Are the class profiles changing? PLoS One 2017; 12: e0182661.

16 French Agency for Food, Environmental and Occupational Health \& Safety (ANSES). Rapport annuel médicaments vétérinaires contenant des antibiotiques en France en 2017. 2018. https://www.anses.fr/fr/system/files/ ANMV-Ra-Antibiotiques2017.pdf.

17 Anonymous. The Swedish Agricultural Administration's regulations on pharmaceuticals and drug use. 2013. http://www.jordbruksverket.se/down load/18.53b6e8e714255ed1fcc4fd9/1385713779939/2013-042.pdf.

18 Borck Hog B, Korsgaard HB, Wolff Sonksen U et al. DANMAP 2016-Use of Antimicrobial Agents and Occurrence of Antimicrobial Resistance in Bacteria from Food Animals, Food and Humans in Denmark. 2017. https://backend. orbit.dtu.dk/ws/portalfiles/portal/140535625/DANMAP_2016_LOW_241017. pdf.

19 Dutch Foundation of the Working Party on Antibiotic Policy (SWAB). NethMap 2015: Consumption of Antimicrobial Agents and Antimicrobial
Resistance among Medically Important Bacteria in the Netherlands/MARAN 2015: Monitoring of Antimicrobial Resistance and Antibiotic Usage in Animals in the Netherlands in 2014. 2016. https://www.wur.nl/upload_mm/6/2/c/ 33cb90db-5bd8-445c-b710-16111aaf369d_NethmapMaran2015.pdf

20 Hansen C. National Average Productivity of Danish Pig Farms 2017. 2018. https://pigresearchcentre.dk/-/media/English-site/english-pdf/1819/Notat_ 1819_UK_CHA.ashx.

21 Anonymous. Antimicrobial Consumption and Resistance in Animals. 2018. https://formularium.amcra.be/.

22 Dupont N, Diness LH, Fertner M et al. Antimicrobial reduction measures applied in Danish pig herds following the introduction of the "Yellow Card" antimicrobial scheme. Prev Vet Med 2017; 138: 9-16.

23 Hawkey PM, Warren RE, Livermore DM et al. Treatment of infections caused by multidrug-resistant Gram-negative bacteria: report of the British Society for Antimicrobial Chemotherapy/Healthcare Infection Society/British Infection Association Joint Working Party. J Antimicrob Chemother 2018; 73: iii2-78.

24 ECDC, European Food Safety Authority, EMA. ECDC/EFSA/EMA Second Joint Report on the Integrated Analysis of the Consumption of Antimicrobial Agents and Occurrence of Antimicrobial Resistance in Bacteria from Humans and Food-producing Animals. 2017. http://doi.wiley.com/10.2903/j.efsa. 2017.4872.

25 Ström G, Boqvist S, Albihn A et al. Antimicrobials in small-scale urban pig farming in a lower middle-income country-arbitrary use and high resistance levels. Antimicrob Resist Infect Control 2018; 7: 35.

26 Moodley A, Nielsen SS, Guardabassi L. Effects of tetracycline and zinc on selection of methicillin-resistant Staphylococcus aureus (MRSA) sequence type 398 in pigs. Vet Microbiol 2011; 152: 420-3.

27 Slifierz MJ, Friendship R, Weese JS. Zinc oxide therapy increases prevalence and persistence of methicillin-resistant Staphylococcus aureus in pigs: a randomized controlled trial. Zoonoses Public Health 2015; 62: 301-8.

28 Slifierz MJ, Friendship RM, Weese JS. Methicillin-resistant Staphylococcus aureus in commercial swine herds is associated with disinfectant and zinc usage. Appl Environ Microbiol 2015; 81: 2690-5.

29 EMA. Questions and Answers on Veterinary Medicinal Products Containing Colistin in Combination with Other Antimicrobial Substances to be Administered Orally. Outcome of a Referral Procedure under Article 35 of Directive 2001/82/EC. 2016. https://www.ema.europa.eu/en/documents/re ferral/questions-answers-veterinary-medicinal-products-containing-colistincombination-other-antimicrobial_en.pdf.

30 Pyörälä S, Baptiste KE, Catry B et al. Macrolides and lincosamides in cattle and pigs: use and development of antimicrobial resistance. Vet $J$ 2014; 200 : 230-9.

31 El Garch F, de Jong A, Simjee S et al. Monitoring of antimicrobial susceptibility of respiratory tract pathogens isolated from diseased cattle and pigs across Europe, 2009-2012: VetPath results. Vet Microbiol 2016; 194: 11-22.

32 Dayao DAE, Gibson JS, Blackall PJ et al. Antimicrobial resistance in bacteria associated with porcine respiratory disease in Australia. Vet Microbiol 2014; 171: 232-5.

33 Klein U, de Jong A, Moyaert Het al. Antimicrobial susceptibility monitoring of Mycoplasma hyopneumoniae and Mycoplasma bovis isolated in Europe. Vet Microbiol 2017; 204: 188-93.

34 FDA. 2016 Summary Report on Antimicrobials Sold or Distributed for Use in Food-Producing Animals. 2017. https://www.cdfa.ca.gov/ahfss/AUS/docs/ FDA_2016_Summary_Report_Antimicrobials_Sold.pdf.

35 More SJ, Clegg TA, McCoy F. The use of national-level data to describe trends in intramammary antimicrobial usage on Irish dairy farms from 2003 to 2015. J Dairy Sci 2017; 100: 6400-13. 
36 de Jong A, El Garch F, Simjee S et al. Monitoring of antimicrobial susceptibility of udder pathogens recovered from cases of clinical mastitis in dairy cows across Europe: VetPath results. Vet Microbiol 2018; 213: 73-81.

37 Suojala L, Kaartinen L, Pyörälä S et al. Treatment for bovine Escherichia coli mastitis - an evidence-based approach. J Vet Pharmacol Ther 2013; 36: 521-31.

$38 \mathrm{SDa}$ Report. Usage of Antibiotics in Agricultural Livestock in the Netherlands in 2016. 2017. https://www.autoriteitdiergeneesmiddelen.nl/en/ news/21/sda-report-usage-of-antibiotics-in-agricultural-livestock-in-thenetherlands-in-2016.

39 Crepieux T, Miller C, Regev-Shoshani G et al. Randomized, non-inferiority trial comparing a nitric oxide releasing solution with a macrolide antibiotic for control of bovine respiratory disease in beef feedlot calves at high-risk of developing respiratory tract disease. Res Vet Sci 2016; 105: 216-21.

40 United States Department of Agriculture. Feedlot 2011 Part IV: Health and health management on U.S. feedlots with a capacity of 1,000 or more head. 2013. https://www.aphis.usda.gov/animal_health/nahms/feedlot/ downloads/feedlot2011/Feed11_dr_PartIV_1.pdf.

41 Baptiste KE, Kyvsgaard NC. Do antimicrobial mass medications work? A systematic review and meta-analysis of randomised clinical trials investigating antimicrobial prophylaxis or metaphylaxis against naturally occurring bovine respiratory disease. Pathog Dis 2017; 75: ftx083.

42 Amachawadi RG, Nagaraja TG. Liver abscesses in cattle: a review of incidence in Holsteins and of bacteriology and vaccine approaches to control in feedlot cattle. J Anim Sci 2016; 94: 1620-32.

43 Lhermie G, Verteramo Chiu L, Kaniyamattam K et al. Antimicrobial policies in United States beef production: choosing the right instruments to reduce antimicrobial use and resistance under structural and market constraints. Front Vet Sci 2019; 6: 245.

44 Devreese M, Antonissen G, De Baere S et al. Effect of administration route and dose escalation on plasma and intestinal concentrations of enrofloxacin and ciprofloxacin in broiler chickens. BMC Vet Res 2014; 10: 289.

45 Hofacre CL, Fricke JA, Inglis T. Antimicrobial drug use in poultry. In: Antimicrobial Therapy in Veterinary Medicine. 5th edn. John Wiley \& Sons, Inc., 2013.

46 Raviv Z, Ley DH. Mycoplasma gallisepticum infection. In: The 13th Edition of Diseases of Poultry. John Wiley \& Sons, 2013; 877-93.

47 EMA. Reflection paper on the use of macrolides, lincosamides and streptogramins (MLS) in food-producing animals in the European Union: development of resistance and impact on human and animal health. EMA/CVMP/ SAGAM/741087/2009. 2011. https://www.ema.europa.eu/en/documents/sci entific-guideline/reflection-paper-use-macrolides-lincosamides-streptogra mins-mls-food-producing-animals-european_en-0.pdf.

48 Glisson JR, Hofacre CL, Mathis GF. Comparative efficacy of enrofloxacin, oxytetracycline, and sulfadimethoxine for the control of morbidity and mortality caused by Escherichia coli in broiler chickens. Avian Dis 2004; 48: 658-62.

49 Scientific Advisory Group on Antimicrobials of the Committee for Medicinal Products for Veterinary Use. Reflection paper on the use of third and fourth generation cephalosporins in food producing animals in the European Union: development of resistance and impact on human and animal health. J Vet Pharmacol Ther 2009; 32: 515-33.

50 Nhung NT, Chansiripornchai N, Carrique-Mas JJ. Antimicrobial resistance in bacterial poultry pathogens: a review. Front Vet Sci 2017; 4: 1-17.

51 Weese JS, Blondeau JM, Boothe D et al. Antimicrobial use guidelines for treatment of urinary tract disease in dogs and cats: Antimicrobial Guidelines Working Group of the International Society for Companion Animal Infectious Diseases. Vet Med Int 2011; 2011: 1-9.

52 Hillier A, Lloyd DH, Weese JS et al. Guidelines for the diagnosis and antimicrobial therapy of canine superficial bacterial folliculitis (Antimicrobial
Guidelines Working Group of the International Society for Companion Animal Infectious Diseases). Vet Dermatol 2014; 25: 1-15.

53 Lappin MR, Blondeau J, Boothe D et al. Antimicrobial use guidelines for treatment of respiratory tract disease in dogs and cats: Antimicrobial Guidelines Working Group of the International Society for Companion Animal Infectious Diseases. J Vet Intern Med 2017; 31: 279-94.

54 Van Cleven A, Sarrazin S, de Rooster H et al. Antimicrobial prescribing behaviour in dogs and cats by Belgian veterinarians. Vet Rec 2018; 182: 324.

55 Buckland EL, O'Neill D, Summers J et al. Characterisation of antimicrobial usage in cats and dogs attending UK primary care companion animal veterinary practices. Vet Rec 2016; 179: 489.

56 Burke S, Black V, Sánchez-Vizcaíno F et al. Use of cefovecin in a UK population of cats attending first-opinion practices as recorded in electronic health records. J Feline Med Surg 2016; 19: 687-92.

57 Singleton DA, Sanchez-Vizcaino F, Dawson S et al. Patterns of antimicrobial agent prescription in a sentinel population of canine and feline veterinary practices in the United Kingdom. Vet J 2017; 224: 18-24.

58 Murphy CP, Reid-Smith RJ, Boerlin P et al. Out-patient antimicrobial drug use in dogs and cats for new disease events from community companion animal practices in Ontario. Can Vet J 2012; 53: 291-8.

59 Escher M, Vanni M, Intorre L et al. Use of antimicrobials in companion animal practice: a retrospective study in a veterinary teaching hospital in Italy. J Antimicrob Chemother 2011; 66: 920-7.

60 Raab O, Béraud R, Tefft KM et al. Successful treatment of Corynebacterium urealyticum encrusting cystitis with systemic and intravesical antimicrobial therapy. Can Vet J 2015; 56: 471-5.

61 Papich MG. Selection of antibiotics for meticillin-resistant Staphylococcus pseudintermedius: time to revisit some old drugs? Vet Dermatol 2012; 23: 352-e64.

62 Spohr A, Schjøth B, Wiinberg B et al. Antibiotic Use Guidelines for Companion Animal Practice. 2012. https:/www.ddd.dk/media/2175/ assembled_final.pdf.

63 Berlin S, Randow T, Scheuch E et al. Pharmacokinetics and pulmonary distribution of gamithromycin after intravenous administration in foals. $J$ Vet Pharmacol Ther 2017; 40: 406-10.

64 Hildebrand F, Venner M, Giguere S. Efficacy of gamithromycin for the treatment of foals with mild to moderate bronchopneumonia. $J$ Vet Intern Med 2015; 29: 333-8.

65 Hughes LA, Pinchbeck G, Callaby R et al. Antimicrobial prescribing practice in UK equine veterinary practice. Equine Vet J 2012; 45: 141-7.

66 Schwechler J, van den Hoven R, Schoster A. Antimicrobial prescribing practices by Swiss, German and Austrian equine practitioners. Vet Rec 2016; 178: 216.

67 Cuny C, Strommenger B, Witte W et al. Clusters of infections in horses with MRSA ST1, ST254, and ST398 in a veterinary hospital. Microb Drug Resist 2008; 14: 307-10.

68 O'Mahony R, Abbott Y, Leonard FC et al. Methicillin-resistant Staphylococcus aureus (MRSA) isolated from animals and veterinary personnel in Ireland. Vet Microbiol 2005; 109: 285-96.

69 Weese JS, Archambault M, Dick $\mathrm{H}$ et al. Methicillin-resistant Staphylococcus aureus in horses and horse personnel, 2000-2002. Emerg Infect Dis 2005; 11: 430-5.

70 Schmiedel J, Falgenhauer L, Domann E et al. Multiresistant extendedspectrum $\beta$-lactamase-producing Enterobacteriaceae from humans, companion animals and horses in central Hesse, Germany. BMC Microbiol 2014; 14: 187.

71 Maddox TW, Clegg PD, Diggle PJ et al. Cross-sectional study of antimicrobial-resistant bacteria in horses. Part 1: Prevalence of antimicrobialresistant Escherichia coli and methicillin-resistant Staphylococcus aureus. Equine Vet J 2011; 44: 289-96. 
72 Weese JS, Lefebvre SL. Risk factors for methicillin-resistant Staphylococcus aureus colonization in horses admitted to a veterinary teaching hospital. Can Vet J 2007; 48: 921-6.

73 Kruse AB, de Knegt LV, Nielsen LR, et al. No clear effect of initiating vaccination against common endemic infections on the amounts of prescribed antimicrobials for Danish weaner and finishing pigs during 2007-2013. Front Vet Sci 2017; 3: 120.

74 Lago A, Godden SM, Bey R et al. The selective treatment of clinical mastitis based on on-farm culture results: I. Effects on antibiotic use, milk withholding time, and short-term clinical and bacteriological outcomes. J Dairy Sci 2011; 94: 4441-56.

75 Wilson BK, Richards CJ, Step DL et al. Beef species symposium: best management practices for newly weaned calves for improved health and wellbeing. J Anim Sci 2017; 95: 2170-82.

76 Rojo-Gimeno C, Postma M, Dewulf J et al. Farm-economic analysis of reducing antimicrobial use whilst adopting improved management strategies on farrow-to-finish pig farms. Prev Vet Med 2016; 129: 74-87. 